\title{
New insight and future perspective of mesothelin-targeted agents in nuclear medicine
}

\author{
Miriam Conte $^{1} \cdot$ Viviana Frantellizzi $^{1}$ (1) $\cdot$ Antonio Matto $^{1} \cdot$ Giuseppe De Vincentis $^{1}$
}

Received: 1 April 2020 / Accepted: 17 July 2020 / Published online: 23 July 2020

(c) The Author(s) 2020

\begin{abstract}
Purpose The aim of this review is to summarize the main applications of mesothelin-targeting agents in the diagnosis of different types of cancers with a brief mention of nuclear magnetic resonance.

Methods The articles taken into account were selected from PubMed, Scopus, and Web of Sciences, including research articles and abstracts that deal with radioimmunotherapy and new tracers for nuclear medicine and radiodiagnosis. Articles that are not in English have been excluded.

Results Mesothelin-targeting agents were the subject of the selected articles in which tracers as ${ }^{64} \mathrm{Cu}$-DOTA-11-25mAb anti MSLN, ${ }^{111}$ In-MORAb-009-CHX-A $"{ }^{89} \mathrm{Zr}$-MMOT0530A, ${ }^{111} \mathrm{In}$-amatuximab, ${ }^{99 \mathrm{~m}} \mathrm{Tc}-\mathrm{A} 1,{ }^{89} \mathrm{Zr}$-AMA, ${ }^{89} \mathrm{Zr}$-amatuximab, ${ }^{64} \mathrm{Cu}$-amatuximab, ${ }^{89} \mathrm{Zr}$-labeled MMOT0530A and ${ }^{89} \mathrm{Zr}$-B3 found application in detection of malignancies that overexpressed mesothelin. Only one article approached magnetic resonance imaging (MRI) diagnosis using superparamagnetic iron oxide nanoparticles linked to anti-mesothelin antibodies. The tracers proved to be highly sensitive in detecting mesothelin positive cells. ${ }^{89} \mathrm{Zr}$-labeled MMOT0530A could also be used to predict the suitability of patients to radioimmunotherapy.

Conclusions Radiolabeled anti-mesothelin antibodies could be crucial as a treatment tool and for predicting the eligibility and the response of the patient to radioimmunotherapy through the study of the expression grade of mesothelin. They can be a relevant tool for pancreatic adenocarcinoma, lung cancer, human epidermoid carcinoma, ovarian cancer, malignant mesothelioma in which mesothelin is widely expressed.
\end{abstract}

Keywords Mesothelin $\cdot$ Radioimmunotherapy $\cdot$ Ab anti-MSLN $\cdot$ Spions

\section{Introduction}

\section{Definition and functions of mesothelin}

Mesothelin (MSLN) is a glycoprotein of $40 \mathrm{KDa}$ located on the surface cellular membrane through a glycosylphosphatidylinositol (GPI) linkage. The precursor of MSLN is a protein of $71 \mathrm{KDa}$ encoded by 2138-bp-long cDNA and it is characterized by 628 amino acids. It is cleaved into two molecules: a $31 \mathrm{kDa} N$-terminal region called megakaryocyte potentiating factor (MPF) and a $40 \mathrm{kDa}$ protein that is MSLN, the most frequent form present on the cellular membrane [3]. Different malignancies express MSLN on

Viviana Frantellizzi

viviana.frantellizzi@uniroma1.it

Department of Radiological Sciences, Oncology and Anatomical Pathology, Sapienza, "Sapienza" University of Rome, Viale Regina Elena, 324, 00161 Rome, Italy their membranes, while its expression is severely limited in healthy cells. Only low levels were described in pleura cells, pericardium and peritoneum cells [18] but also on the epithelium of the trachea [5], on Fallopian tubes epithelial cells [39], on the surface epithelium of the ovary, on the tunica vaginalis, the rete testis and tonsil [8,39]. Conversely, there are many examples of cancers that overexpress MSLN: gastrointestinal cancers such as adenocarcinoma of the pancreas, ampulla of Vater adenocarcinoma, cholangiocarcinoma, pancreatic ductal adenocarcinoma; also genital cancer are mesothelin-positive as endometrium adenocarcinoma and ovarian non-mucinous carcinoma [9]. Other malignancies as adenocarcinoma of the liver and lungs, the epithelial component of biphasic synovial sarcomas and mesothelioma also express MSLN [39, 49]. The biological function of MSLN is not well understood. It is hypnotized that MSLN was involved in the metastatic spread of mesothelin-bearing cancer cells via its interaction with the protein carbohydrate antigen CA125/mucin 16 
(MUC16) [22]. CA125 is a tumor antigen used for diagnosis and follow-up of ovarian cancer [4, 43, 50, 59]. High levels of CA125 is expressed not only on the cell surface but it can be released in extracellular space in soluble proteolytic fragments form. As CA125 belongs to the mucin family of glycoproteins, it is also called mucin 16 or MUC16 [59]. In particular, the binding between CA125 and MSLN promotes ovarian cancer metastases facilitating their heterotypic cellular adhesion (CA125 itself inhibits the human natural killer cells cytolytic responses), [26, 42], pancreatic cancer cell motility and then invasion into other tissues via matrix metalloproteinase (MMP)-7 activation [6, 53]. Blocking the interaction between MSLN and CA125 with antibodies antiMSLN, the heterotypic cell adhesion has been hindered [50]. Several studies (Table 1) indicate that MSLN expression is correlated with chemoresistance and shorter progressionfree survival and overall survival [7] given by the upregulation of anti-apoptotic proteins as Bcl-2 and Mcl-1 [53]. Its high expression, as recently observed, is correlated with poor prognosis in patients suffering from advanced serous ovarian cancer (SOD) [58]. The review aims to summarize the main uses of labeled MSLN antibodies in diagnosis also briefly citing the recent application in magnetic resonance imaging.

\section{Diagnosis with radiolabeled anti-mesothelin antibodies: an overall view}

Clinical evidence suggests that MSLN could be considered a crucial target for the cancer treatment of MSLN positive tumors. Numerous clinical trials for MSLN-targeting methods gave promising results, as targeting MSLN with antibodies, antibody derivatives, antibody-drug conjugates, immunotoxin and CAR-T cells for therapy (Table 2). To give some examples, in a phase I trial of SS1P, an anti- MSLN immunotoxin, patients with mesotheliomas, ovarian and pancreatic cancer were enrolled and among the 33 treated patients, minor responses were seen in four patients, 19 had a stable disease whereas the progressive disease was seen in ten patients [20,53]. Analogously, a vaccination with autologous mesothelin-positive tumor cells induced a cytotoxic $\mathrm{T}$ cell response to mesothelin epitopes HLA-A2, A3, A2A in three patients who developed a delayed-type hypersensitivity after vaccination. It was evident that a cross-presentation by immunotherapy induced CD8 $\mathrm{T}$ cell responses that are tumor-specific and capable of eradicating cancer [57]. To give another example, it has been proposed to use MSLN -targeted thorium-227 $\left({ }^{227} \mathrm{Th}\right)$ to evaluate survival benefit of alpha therapy for MSLN positive cancers. BAY2287411, a human IgG1 antibody BAY86-1903 (Anetumab) covalently bound to ${ }^{227} \mathrm{Th}$ complexing 3,2-HOPO chelator was used. The agent had a potent in vitro and in vivo activity in models of breast, lung, ovarian and pancreatic cancer derived from cellular patient xenograft (PDX) $[15,17]$. Studies like these show how the detection of MSLN positive cancer cells is crucial to develop targeting tailored therapies and to gain more personalized treatments [10].

\section{Mesothelin and pancreatic cancer}

MSLN is also over-expressed in up to $86-100 \%$ of pancreatic ductal adenocarcinoma (PDAC) [35]. The differences of distribution of MSLN between PDAC and normal tissue were highlighted in a latest study through a standardized immunohistochemical detection with two anti-MSLN antibodies (EPR4509 and EPR19025-42). MSLN seems to be widely distributed on PDAC cell membranes while paracancer tissues had no expression of MSLN with a statistically significant difference $\left(\chi^{2}=40.615, p=0.000\right)$. PDAC represents the fourth most frequent cause of death caused by cancer worldwide and has a 5-years overall survival that is less than $8 \%$. Recent projections show a twofold increase in the PDAC incidence in the U.S. and Europe within the next ten years [40, 47, 48, 52]. New treatments and the development of new sensitive approaches in diagnosis could therefore be an increasingly urgent need. In a clinical phase I trial the MSLN antibody MMOT0530A has been labeled with ${ }^{89} \mathrm{Zr}$ and administered to 11 IV stage patients. Among these, seven had PDAC and four ovarian cancer. In all patients, this method allowed the visualization of at least one lesion. The mean standardized uptake value (SUVmax) of the radiopharmaceutical in 37 lesions was $13.1( \pm 7.5)$ on Positron Emission Tomography (PET) 4 days post-injection. In particular, $11.5( \pm 7.5)$ in $(N=17)$ pancreatic and 14.5 $( \pm 8.7)$ in $(N=20)$ ovarian cancer lesions [31]. Moreover, a single photon emission computed tomography (SPECT) radiopharmaceutical developed has been ${ }^{111}$ In-labeled amatuximab. Of six patients enrolled in the trial, two had PDAC and four malignant mesothelioma. At the imaging, it was possible to recognize at least one tumor lesion from the background. In all patients, the tumor to background ratios (TBR) were 1.2 or superior (range 1.2-62.0), with higher TBR in mesothelioma than pancreatic adenocarcinoma. The uptake was evident in primary tumors but also in metastases [2, 24, 29, 30, 34, 41]. Montemagno et al. proved that high expression of MSLN gene in PDAC patients correlates with a decreased overall survival in comparison with patients with lower expression [38]. In this study, Technetium-99 m $\left({ }^{99 \mathrm{~m}} \mathrm{Tc}\right)-\mathrm{A} 1$, an anti- MSLN single domain antibody labeled with ${ }^{99 \mathrm{~m}} \mathrm{Tc}$, was evaluated in vitro and in vivo. They used high-mesothelin-expressing AsPC-1 cells and moderatemesothelin-expressing SW1990 cells and developed a mouse model of PDAC (AsPC-1). They observed a 2.1-fold higher uptake in AsPC-1 cells than in SW1990 cells. As previously stated, the normal tissue exhibits the glycoprotein MSLN too but it is limited in healthy cells $[18,28,39]$. For this 


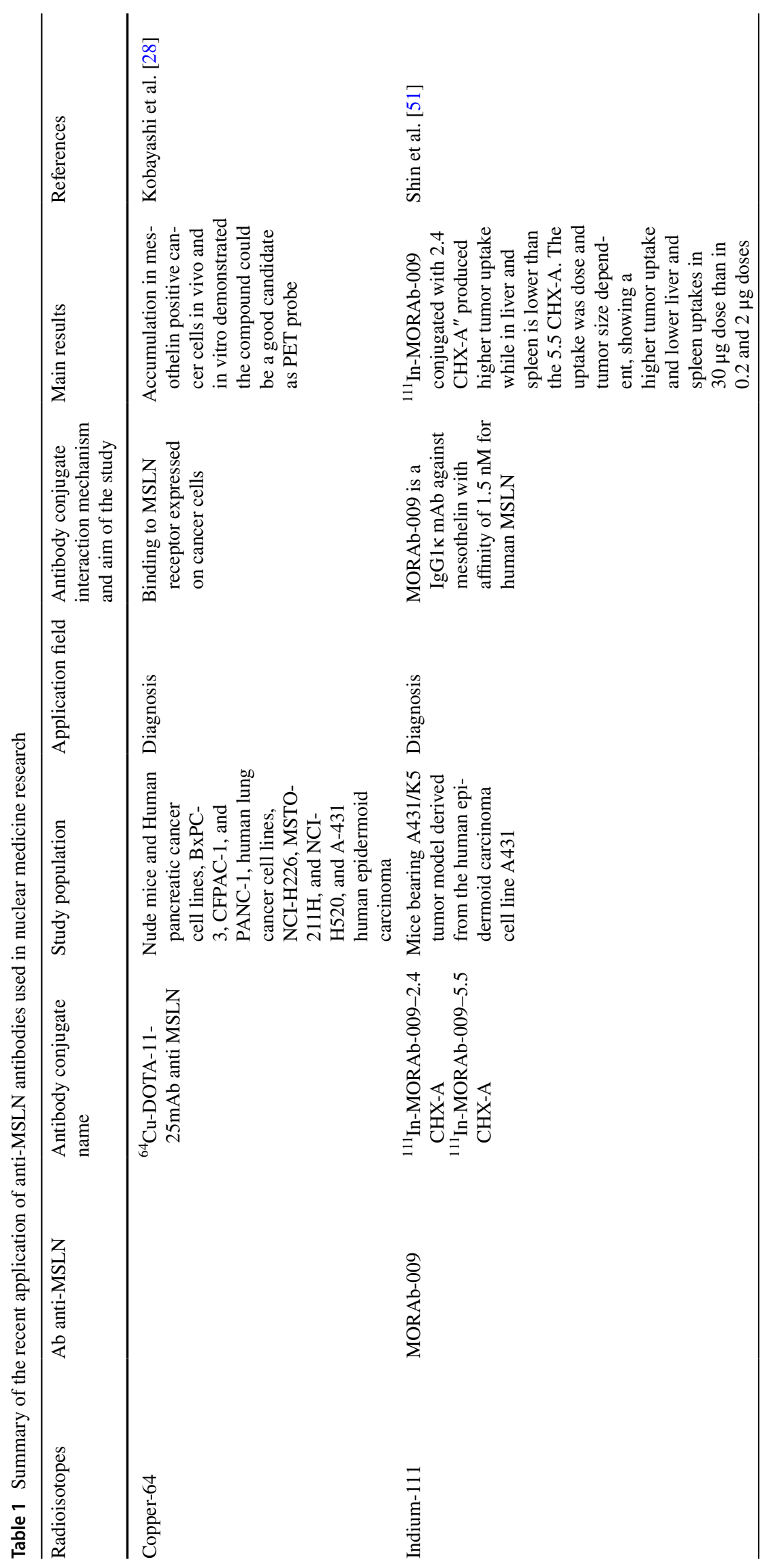




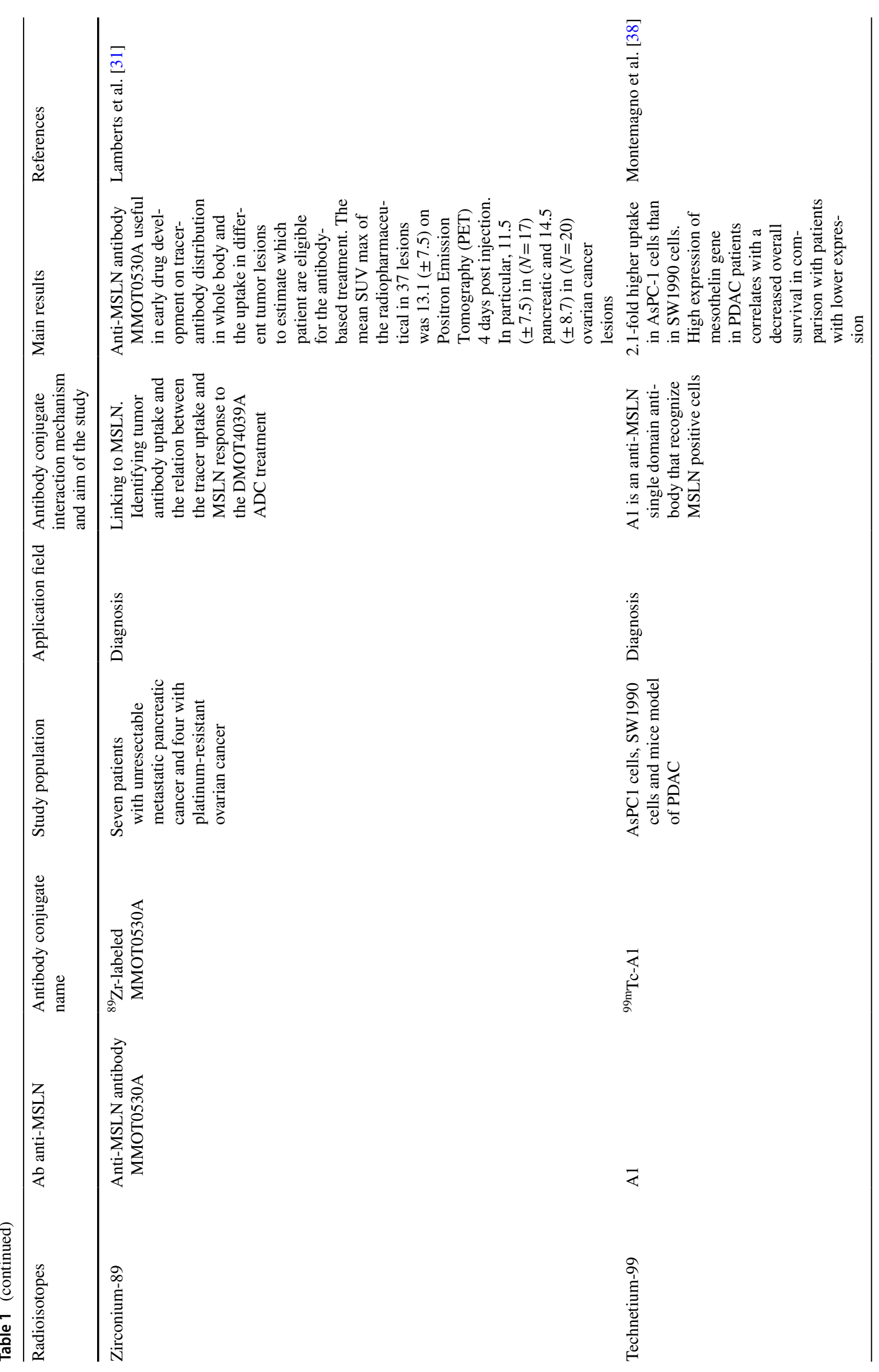




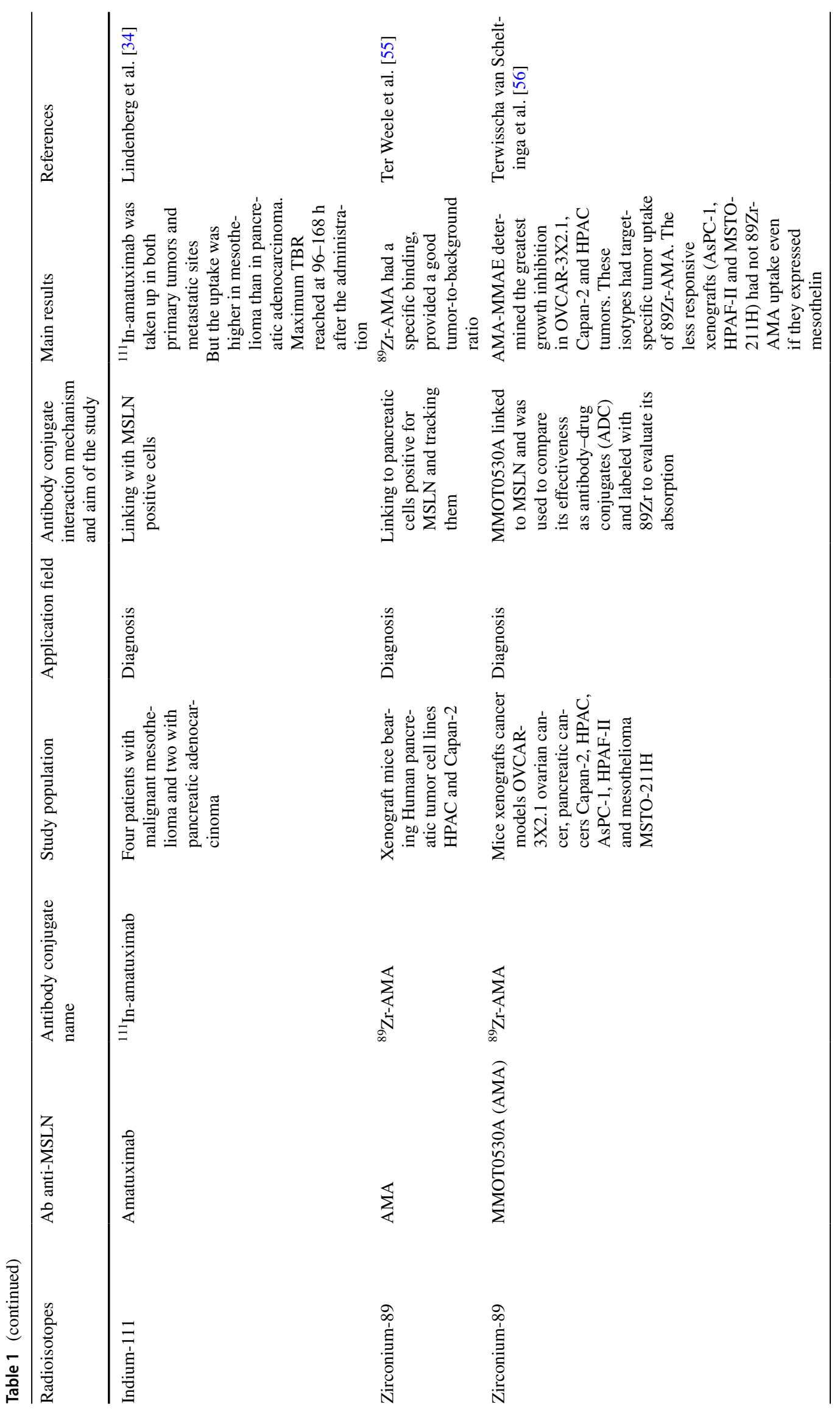




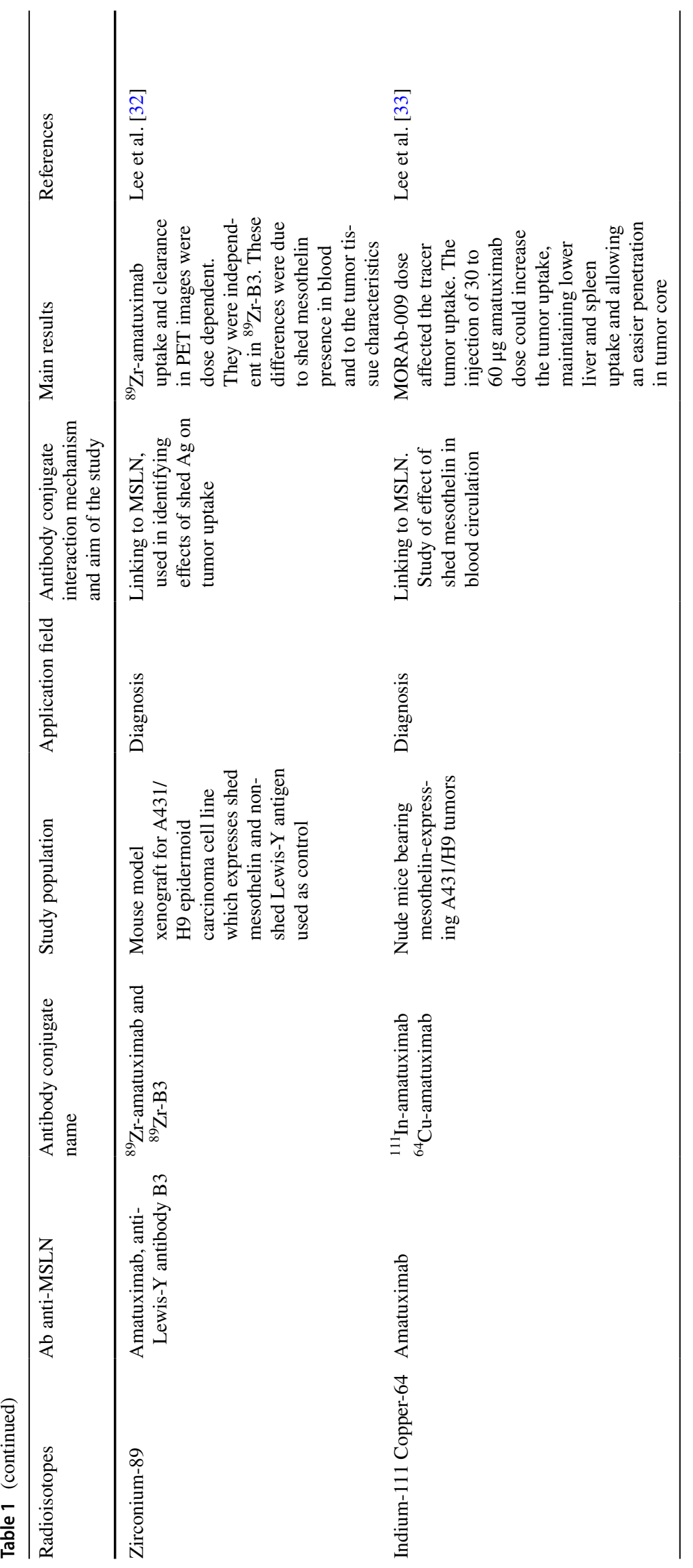




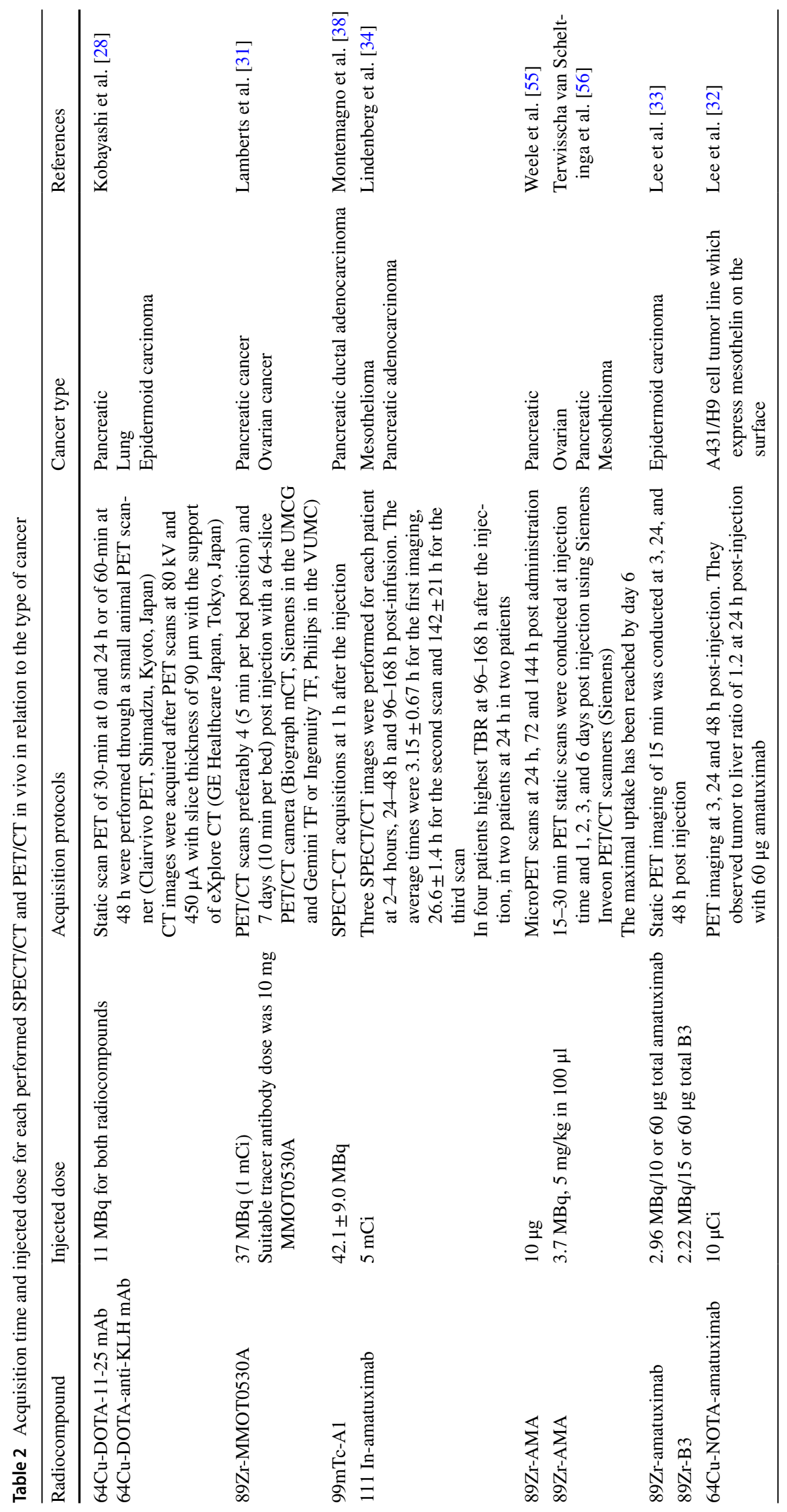


pivotal characteristic, Kobayashi et al. synthesized a hybridoma encoding monoclonal antibody (mAb, clone 11-25) against human MSLN using five-week-old male BALB/c nude mice. Their research aimed to find a suitable target for the development of a diagnostic probe for pancreatic cancer. They valued the presence of MSLN in sera of patients with mesothelioma and found that 11-25 mAb binds to MSLN, not only in a soluble form but was also attached on the membrane. They labeled DOTA-conjugated 11-25 mAb with positron-emitting Copper-64 $\left({ }^{64} \mathrm{Cu}\right)$. Nude mice with human pancreatic cancer xenografts underwent PET imaging. An analog study was conducted in vitro using Human pancreatic cancer cell lines, BxPC-3, CFPAC-1, and PANC-1, human lung cancer cell lines, NCI-H226, MSTO-211H, and NCI$\mathrm{H} 520$, and a human epidermoid carcinoma, A-431. ${ }^{64} \mathrm{Cu}-$ labeled 11-25 mAb accumulation in MSLN positive cancer cells was observed both in vivo and in vitro, proposing it as a good candidate as PET probe. It could be helpful to be used for various types of malignancies and possibly being more specific than Fluorine-18 fluorodeoxyglucose $\left({ }^{18} \mathrm{~F}-\mathrm{FDG}\right)$ that has the pitfall of nonspecific accumulation into the inflammatory lesions. Another radioisotope applied in the research for a new radiotracer for mesothelin is Zirconium-89 $\left({ }^{89} \mathrm{Zr}\right)$. Ter Weele et al. labeled ${ }^{89} \mathrm{Zr}$ with an anti-mesothelin antibody ( ${ }^{89} \mathrm{Zr}$-AMA) for tracking of pancreatic cancer cells in mice [55]. For this aim, they used xenograft mice bearing Human pancreatic tumor cell lines (HPAC) and Capan-2. The nonspecific ${ }^{111} \mathrm{In}$-IgG was compared with ${ }^{89} \mathrm{Zr}$-AMA to evaluate the biodistribution of the radiolabeled compound. The uptake was expressed in terms of SUVmax. For HPAC tumors SUVmax increased from $1.09 \pm 0.24(24 \mathrm{~h})$, to $1.51 \pm 0.29(72 \mathrm{~h})$, and $1.68 \pm 0.33(144 \mathrm{~h})$ after the injection. For Capan-2 tumors uptake increased from $0.70 \pm 0.18$ $(24 \mathrm{~h})$, to $1.18 \pm 0.32(72 \mathrm{~h})$, and $1.40 \pm 0.41(144 \mathrm{~h}){ }^{89} \mathrm{Zr}-$ AMA uptake was also evident in ex vivo studies conducted on tumoral cell lines. ${ }^{89} \mathrm{Zr}$-AMA had a specific binding and provided a good tumor-to-background ratio, so giving important information on the accessibility of the tumor by antibodies and the availability of the target in tumors. It could be proposed as the Antibody-drug conjugates treatment tool, as this therapeutic efficacy is dependent on the accessibility, the representation and the internalization in the tumor target [55].

\section{Radiolabeled MORAb-009}

MORAb-009 (amatuximab) is an IgG1к mAb (monoclonal antibody) against MSLN with an affinity of $1.5 \mathrm{nM}$ for human MSLN [22]. Moreover, as shown in the paper by Shin et al. it has been linked to 2-(pisothiocyanatobenzyl)cyclohexyl-diethylenetriamine-pentaacetic acid (CHX-A") and labeled with ${ }^{111}$ In [51]. The distribution of the compound has been studied using a mouse bearing A431/K5 tumor model to establish if this molecule could be useful in the diagnosis and the therapy. A431/K5 is a specific type of cell line derived from the human epidermoid carcinoma cell line A431. The cells have been obtained by the cotransfection of human epidermoid cancer A431 cells with a plasmid that encodes for MSLN and a neomycin resistance gene. They are injected in the right hind flank of athymic mice which developed cancer. The biodistribution studies of MORAb-009 showed an increased concentration of the antibody in the MSLN positive cancer cells. The tumor uptake was MSLN-mediated. It was demonstrated by the 4-time higher uptake in A431/K5 tumor than in A431 tumor cells. In particular, the $2.4 \mathrm{CHX}$-A conjugated with ${ }^{111} \mathrm{In}$ MORAb-009 produced higher tumor uptake while in liver and spleen it is lower than the 5.5 CHX-A. The uptake is dependent on the MORAb-009 dose and tumor size, showing a higher tumor uptake and lower liver and spleen uptakes in $30 \mu \mathrm{g}$ dose than in 0.2 and $2 \mu \mathrm{g}$ doses. Hassan et al. studied MORAb-009 and concluded that MORAb-009 in combination with chemotherapy caused a marked reduction in tumor growth of ${ }^{{ }}$-expressing tumors in nude mice. Similar results could not be reached by chemotherapy or MORAb009 treatment alone [22]. The achievements obtained by Shin et al. consequently, add other pivotal aspects that affect future utilization of MORAb-009. It is crucial to consider parameters as the number of chelate conjugation and the injected dose to obtain high tumor uptake and reduce nontarget organ uptake of ${ }^{111}$ In labeled MORAb-009.

In another study, after the treatment with MORAb-009, an increase in serum CA-125 was evident. The increase of CA-125 was not due to a progression disease. After stopping the MORAb-009 treatment, the serum levels of CA-125 decreased. The increase of CA-125 during the treatment seemed produced by the inhibiting capacity of MORAb-009 towards the binding of tumor shed CA-125 to MSLN on mesothelial cellular membranes. The inhibition of the interaction between MSLN and CA-125 could also be an interesting therapy tool to hinder tumor metastases in mesotheliomas and ovarian malignancies [25]. In addition, it was demonstrated that MORAb-009 is well tolerated with a maximum tolerated weekly dose (MTD) of $200 \mathrm{mg} /$ $\mathrm{m}^{2}$ [21]. A significant study was conducted by Lindenberg et al. that studied the biodistribution and dosimetry of amatuximab radiolabeled with ${ }^{111} \mathrm{In}$ in MSLN positive cancers $[23,34]$. Amatuximab is also used in mesothelioma cancer therapy. They enrolled seven patients, four with malignant mesothelioma and two with pancreatic adenocarcinoma. All the patients underwent SPECT/CT after administration of ${ }^{111}$ In amatuximab except for one patient, unable to lie on the scanner. The SPECT/CT images were obtained after 2-4 h, 24-48 $\mathrm{h}$ and 96-168 $\mathrm{h}$ after the injection and TBR was of 1.2 (range 1.2-62.0). ${ }^{111}$ In-amatuximab was taken up by both primary tumors and metastatic sites but 
this uptake was higher in mesothelioma than in pancreatic adenocarcinoma. Heart, liver, kidneys, and spleen were the physiological sites of uptake [54]. ${ }^{111}$ In amatuximab reached the maximum TBR at $96-168 \mathrm{~h}$ after the injection. Before $96 \mathrm{~h}$, the non-specific uptake of the background hinders the visualization of tumor uptake.

\section{Diagnosis for antibody-drug conjugates treatment eligibility}

An exciting field is represented by antibody-drug conjugate (ADC). ADC is a specific mAb linked with a cytotoxic drug. The acting mechanism is its release after internalization in the intracellular environment acting as a carrier to permit the internalization of potent cytotoxic drugs to tumor cells expressing the substrate for the specific antibody. Recently Hassan et al. [19] conducted a multicenter phase I study to establish the safety and the tolerability of anetumab ravtansine (BAY 94-9343), an ADC conjugated to the human immunoglobulin G1 anti-MSLN monoclonal antibody linked to the maytansine derivative tubulin inhibitor DM4. They enrolled 148 adult patients with multiple solid tumor types. Among these, ten patients were included in a dose-escalation study receiving a dose of $0.15-7.5 \mathrm{mg} / \mathrm{kg}$ anetumab ravtansine once every 3 weeks. They administered to the six patients with an advanced, recurrent ovarian cancer or a malignant mesothelioma a dose of $1.8 \mathrm{mg} / \mathrm{kg}$ once per week and $2.2 \mathrm{mg} / \mathrm{kg}$ once per week anetumab ravtansine once every 3 weeks. The study highlighted that the maximum tolerated dose of the ADC was $6.5 \mathrm{mg} / \mathrm{kg}$ once every 3 weeks or $2.2 \mathrm{mg} / \mathrm{kg}$ once per week. The side effects were fatigue, nausea, diarrhea, anorexia, vomiting, peripheral sensory neuropathy, and keratitis/keratopathy while related-drug deaths were not evidenced. These results demonstrated BAY 94-9343 safety, tolerability, and manageability in patients with advanced or metastatic solid tumors, including mesothelioma and ovarian cancer that was refractory to standard therapy. Common adverse events moreover were manageable with treatment interruptions or dose reductions. However, even though the encouraging results, the authors specified that all patients who responded to ADC had high MSLN expression tumors, some patients had at least $60 \%$ tumor MSLN expression but did not respond to BAY 94-9343. Further investigations are required to explore the correlation between MSLN expression and the response to the treatment also including other malignancies. Two other examples of a molecule that acts in this way are monomethyl auristatin E (MMAE) and maytansinoid drug conjugates, which is brentuximab vedotin for CD30-positive lymphomas and trastuzumabemtansine (TDM1) for metastatic breast cancer. An anti- MSLN antibody, MMOT0530A, was used to compare its effectiveness as ADC. It has been labeled with ${ }^{89} \mathrm{Zr}$ to evaluate imaging tumor absorption $[13,56]$. The inhibition of MSLN -targeted tumor growth after the administration of MMAE,
ADC AMA-MMAE (DMOT4039A), was measured in mouse xenograft cancer models: OVCAR-3 X2.1 ovarian cancer, pancreatic cancers Capan-2, HPAC, AsPC-1, HPAF-II and mesothelioma MSTO-211H. The humanized IgG1 5B6, an antibody to HSV-1 viral coat protein $\mathrm{gD}$, was used as nonbinding isotype control (anti-gD) and was linked to MMAE (antigD-MMAE). Tumor growth inhibition after the administration of ADC was compared with the tumor antibody uptake using the ${ }^{89} \mathrm{Zr}$-labeled AMA. AMA-MMAE determined the greatest growth inhibition in OVCAR-3X2.1, Capan-2 and HPAC tumors. These isotypes had target-specific tumor uptake of ${ }^{89} \mathrm{Zr}$-AMA. The less responsive xenografts (AsPC-1, HPAF-II, and MSTO-211H) did not have ${ }^{89} \mathrm{Zr}$-AMA uptake even if they expressed MSLN. It was an interesting result giving the basis for a new perspective of treatment effects of the AMA-MMAE therapeutic ADC prediction. Nevertheless, absolute tumor absorption of ${ }^{89} \mathrm{Zr}$-AMA is not necessarily predictive for the effectiveness of ADC. Many factors are important for the outcome. Examples are cellular sensitivity to the drug, the operation of drug efflux pumps or other resistance mechanisms, changes in vascular density and permeability, the nature of the extracellular matrix and the presence of non-internalizing soluble MSLN (Fig. 1) [56]. Lamberts et al. selected a study population of seven patients with unresectable metastatic pancreatic cancer that was histologically confirmed and four with platinum-resistant ovarian cancer. These patients had been included in the phase I study with DMOT4039A, an ADC formed by the humanized IgG1 mAb MMOT0530A and the mitotic agent monomethyl auristatin MMAE. The study aimed to identify tumor antibody uptake and the relation between the tracer uptake and MSLN response to the ADC treatment. ${ }^{89} \mathrm{Zr}$-labeled anti-MSLN antibody MMOT0530A exhibited yet a specific tumor uptake on microPET at 1, 3 and 6 days after the injection of tracer yet in human MSLN positive tumorbearing mice $[31,55]$. In the study of Lamberts et al. $37 \mathrm{MBq}$ ${ }^{89} \mathrm{Zr}$-labeled anti-MSLN MMOT0530A was administered in all patients and after 2, 4, and 7 days post-injection the patients underwent to PET/CT imaging and the uptake was expressed as SUV. The convenient dose was $10 \mathrm{mg}$ MMOT0530A with an improved visualization in the imaging at 4 and 7 days postinjection. 37 lesions has a mean SUVmax of $13.1( \pm 7.5)$ on 4 days post-injection PET, with $11.5( \pm 7.5)$ in $(N=17)$ pancreatic and $14.5( \pm 8.7)$ in $(N=20)$ ovarian cancer lesions. The uptake visualized in blood, liver, kidneys, spleen, and intestine was attributable to the normal antibody distribution. The best response to DMOT4039A was in one patient that showed a partial response. They concluded that this tracer could give information in early drug development on tracer-antibody distribution in the whole body and their uptake in different tumor lesions to estimate which patients are eligible for the antibodybased treatment. The ZEPHIR study [16] demonstrated the predictive value of pretreatment with ${ }^{89} \mathrm{Zr}$-trastuzumab PET in patients with metastatic breast cancer before they received 
Fig. 1 Represents coronal views in mouse at maximum projection PET scan. In image a,

${ }^{89} \mathrm{Zr}$-AMA images and control

$\left({ }^{89} \mathrm{Zr}-\mathrm{gD}\right)$ antibodies in ovarian cancer OVCAR-3X2.1; Capan-2 pancreatic cancer in image $\mathbf{b}$; HPAC pancreatic cancer in $\mathbf{c}$; AsPC-1 pancreatic cancer in image $\mathbf{d}$ and HPAF-II pancreatic cancer in e; mesothelioma MSTO211H in image f. Arrows indicate the tumor on the mice flank and the response to DMOT4039A in the different tumor types. On the left the tumor uptake of the radiotracer, on the right the control [56]
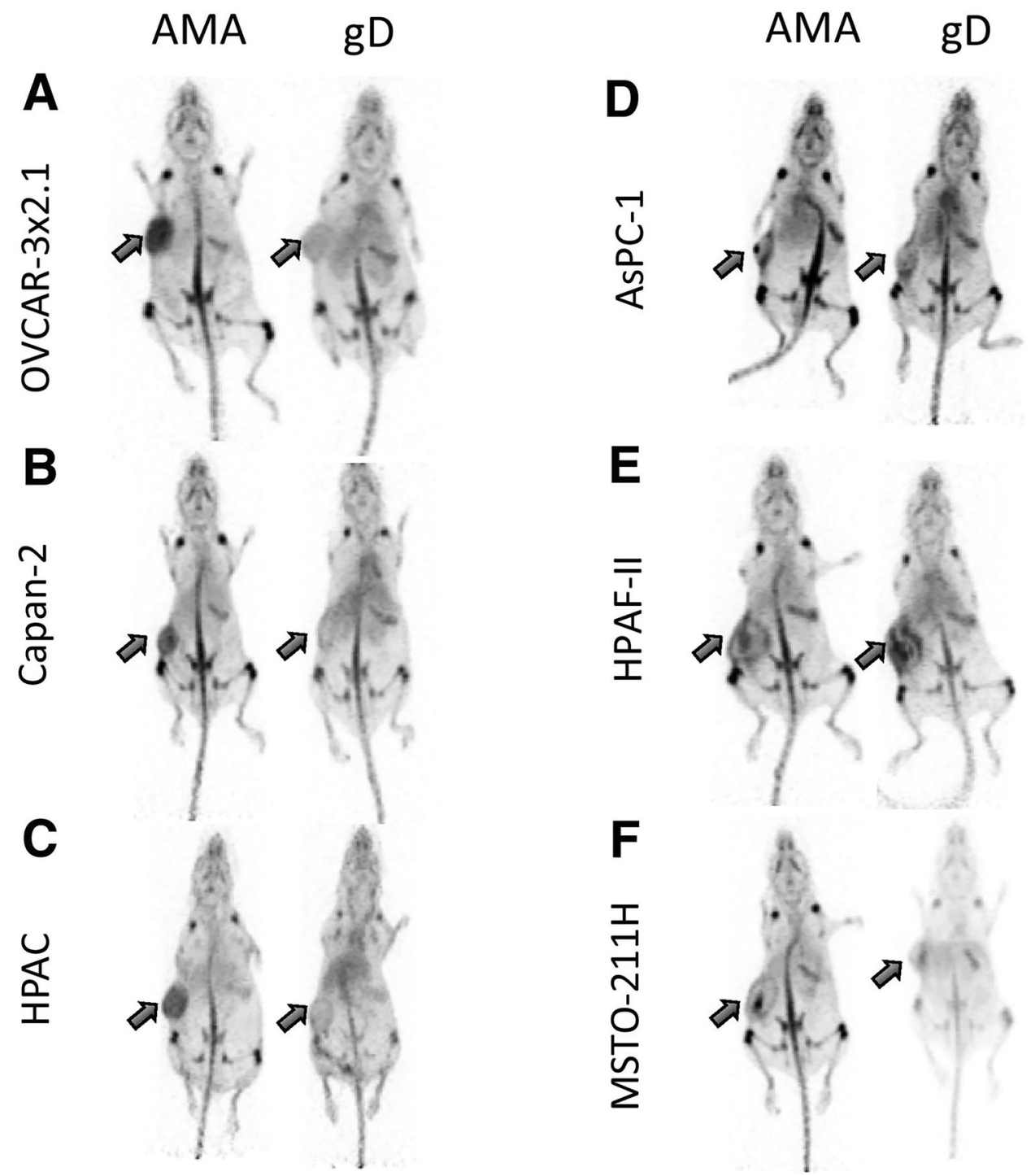

ADC TDM1 treatment. Otherwise, using ${ }^{89} \mathrm{Zr}$-trastuzumab PET in combination with an early ${ }^{18} \mathrm{~F}$-FDG PET demonstrated a negative predictive value for RECIST response of $100 \%$. This proved that combining these two techniques could be a potentially helpful tool to select the patient that could respond to ado-trastuzumab emtansine (T-DM1). Analogously, ${ }^{89} \mathrm{Zr}$ MMOT0530A PET may be a promising tracer in combination with DMOT4039A to identify a patient that responds to the treatment with DMOT4039A [31].

\section{Pitfalls of mesothelin use: effects of shed mesothelin on antibodies binding capacity}

A limit of MSLN use is the presence in the bloodstream of high levels of shed antigen that hamper the capability of the $\mathrm{mAb}$ in biding tumor antigen [27, 44]. Pimm et al. in particular, noticed that the immune complex formed by $\mathrm{mAb}$ and tumor antigen in mice with colon carcinoma xenografts had a hepatic localization of the radioiodinated monoclonal antibody due to its hepatic clearance [44, 45]. To identify the effects of shed Ag on tumor uptake, two antibodies, anti-MSLN mAb amatuximab, and control anti-Lewis-Y mAb B3, were compared [33]. The population study has been constituted by a mouse model bearing A431/H9 tumor expressing both shed MSLN and noshed Lewis-Y antigen. At $24 \mathrm{~h}$ the tumor, hepatic, splenic uptake and the presence in bloodstream of ${ }^{89} \mathrm{Zr}$-amatuximab was directly correlated with dose levels. Oppositely, the uptake of anti-Lewis-Y antibody ${ }^{89} \mathrm{Zr}$-B3 in these districts was dose-independent. ${ }^{89} \mathrm{Zr}$-amatuximab tumor uptake was evident in PET at $3 \mathrm{~h}$ post-injection (p.i.) at 10 and $60 \mu \mathrm{g}$ dose levels. During the 3-48 h period, ${ }^{89} \mathrm{Zr}$-amatuximab was washed out from the blood at the $10 \mu \mathrm{g}$ dose while at the $60 \mu \mathrm{g}$ dose ${ }^{89} \mathrm{Zr}$ amatuximab gradually cleared from the bloodstream and a drastic increase in tumor uptake (\%ID/g) was evident at $24 \mathrm{~h}$ and 48 h. ${ }^{89} \mathrm{Zr}-\mathrm{B} 3 \mathrm{had}$ tumor uptake at $3 \mathrm{~h}$ p.i. at 15 and 
$60 \mu \mathrm{g}$ doses. But, opposite to ${ }^{89} \mathrm{Zr}$-amatuximab PET images, the uptake and the clearance of ${ }^{89} \mathrm{Zr}-\mathrm{B} 3$ was not influenced by dose effects. These differences in biodistribution characteristics may be explained by the shed MSLN presence in blood and also by the tumor tissue that influences the serum half-life and the Ag-specific tumor uptake of radiolabeled amatuximab [33]. The effect of shed MSLN in blood circulation has already been previously defined in a study conducted on nude mice bearing the A431/K5 tumor: the tumor targeting of amatuximab labeled with ${ }^{111} \mathrm{In}$ or ${ }^{64} \mathrm{Cu}$ decreased in the presence of shed MSLN in the bloodstream. The spleen and liver uptakes increased. The blood retention and tumor uptake diminished when the dose was unable to saturate the shed MSLN. It was noted that the MORAb-009 dose affected the tumor uptake: a $30-\mu \mathrm{g}$ dose was correlated to a higher tumor uptake than the $0.2-\mu \mathrm{g}$ and $2-\mu \mathrm{g}$ doses but it determined lower liver and spleen uptakes than the 0.2$\mu \mathrm{g}$ dose [51]. The injection of 30-60 $\mu \mathrm{g}$ amatuximab dose could increase the tumor uptake, maintaining lower liver and spleen uptake and allowing an easier penetration in the tumor core $[32,51]$.

\section{SPIONs and mesothelin}

A recent prospective in diagnosis is using superparamagnetic iron oxide nanoparticles (SPIONs), $20-150 \mathrm{~nm}$ crystals of iron oxide with a functionalized shell that gains for the particles more stability in water and different biochemical properties for many uses in the biochemical field [14]. Shao et al. thought to bind SPIONs with Ab anti-mesothelin (A-MSF) to detect pancreatic carcinoma cells using mouse xenografts. The nanoparticle shell was constituted by silica that gave good biocompatibility to the compound, called $\mathrm{Fe}_{3} \mathrm{O}_{4} @ \mathrm{SiO}_{2}$ (FS). A-MSF has a T2 relaxation rate of $59.435 \mathrm{nM} / \mathrm{s}$ and $\mathrm{T} 1$ of $0.549 \mathrm{nM} / \mathrm{s}$. After the injection of the probe FS linked to A-MSF, a decrease of the tumor signal was observed demonstrating that the compound could be a valid T2 targeting agent in pancreatic cancer of nude mice [35].

There are different types of SPIONs based on dimension differences: oral SPIONs have a diameter between $300 \mathrm{~nm}$ and $3.5 \mu \mathrm{m}$, standard SPIONs size is $50-150 \mathrm{~nm}$ and ultra-small SPIONs have diameter smaller than $50 \mathrm{~nm}$ $[1,12,14]$. In a study of Deng et al. ultrasmall superparamagnetic iron oxides (USPIOs) were also linked to antimesothelin antibody-conjugated PEGlyated liposomal doxorubicin (M-PLDU). M-PLDUs obtained were spherical with a diameter about $\sim 180 \mathrm{~nm}$ and a zeta potential of about $-28 \sim-30 \mathrm{mV}$. Panc-1 human pancreatic cancer carcinoma cell line for in vitro antitumor activity evaluation was used. The half-maximal inhibitory concentration values were $0.53 \mu \mathrm{M}$ for free DOX, 1.95 for M-PLDUs, and 3.5 for PEGlyated nanoimmunoliposome without antibody conjugation (PLDUs). These results showed that M-PLDU has an antitumor activity 1.8 times higher than PLDU. No significant cytotoxicity was determined by USPIOs. To establish the antitumor activity in vivo and to evaluate the anticancer effect of M-PLDU in the animal model, BALB/c $\mathrm{nu} / \mathrm{nu}$ mice bearing Panc-1 xenografts $\left(\sim 50-60 \mathrm{~mm}^{3}\right)$ were injected intravenously with FD, PLDUs and M-PLDUs once a week. The total DOX dosage administered was equal to $6 \mathrm{mg} / \mathrm{kg}(2 \mathrm{mg} / \mathrm{kg})$. The antitumor effect of M-PLDUs was confirmed by the final tumor weigh in the animals: for M-PLDUs it was $0.06 \mathrm{~g}$ versus $0.10 \mathrm{~g}$ in the mice treated with PLDUs and $0.18 \mathrm{~g}$ in FD treatment. After $4 \mathrm{~h}$ from the injection, RMN showed the dropping of the tumor signal intensity (SI), where the lower SI demonstrated the higher concentration of iron oxide in the tissue. M-PLDUs demonstrated to be an affective delivery system to vehicle anticancer drugs but also a promising contrast agents to pancreatic cancer cells [11]. Another solution for immunotargeting against tumor antigens has been binding Biobodies $(\mathrm{Bb})$ with SPIONs. Biobodies are fragments of antibodies that are biotinylated by yeast and secreted into the culture environment. In the research of Prantner et al. Bb against MSLN were obtained and linked to streptavidin-labeled nanoparticles (SA-SPIONs). Chemical-based strategies used for bioconjugating antibodies to nanoparticles have the deal to produce heterogeneous populations of antibodynanoparticle conjugates with different number and site of covalent bond formation. It can lead to a damage in the site of antibody binding conformation and inhibit the recognition of its antigen. SA-SPIONs, on the contrary, permit a selected and rapid self-assembly of immunotargeted nanoparticles in the yeast culture supernatant thanks to the high sensibility and specificity of the binding between Bb and SA-SPIONs. Even if it is necessary to conduct further investigation, $\mathrm{Bb}$ SA-SPIONs represent a step forward in research of more and more specific therapies that can spare normal tissue limiting the systemic side-effects of nowadays chemotherapies [46]. Recently, dual-modality SPECT/MR imaging agents for SPECT/MRI scanners have been proposed with the aim to achieve a dual modality molecular imaging bioprobes for MSLN positive cancer. 111In labelled anti-mesothelin antibody mAbMB (111In-mAbMB), yet proposed as SPECT probes [37], has been conjugated with SPIONs. $74 \mathrm{kBq}$ of 111InmAbMB-SPIONs were administered to three SCID mice bearing A431K5 tumor xenografts. A431K5 cells are human epidermoid A431 cells transfected with the plasmid pcD3CAK1-9 which encodes and produces the MSLN. After 24 and $72 \mathrm{~h}$ the mice were underwent $7 \mathrm{~T} \mathrm{MR}$ imaging. The results demonstrated specific uptake into A431K5 tumors and a change of the SI was seen in MRI, as a hypointensity area, in correspondence with cancer localization and confirmed through autoradiographs [36]. 


\section{Conclusions}

MSLN represents an intriguing subject of research. Different oncological applications have been explored, such as mesothelioma, ovarian adenocarcinoma, lung cancer, human epidermoid carcinoma to PDA and colon adenocarcinoma, as a consequence of the variety of MSLN expression in tumors. ${ }^{64} \mathrm{Cu}$-DOTA-11-25mAb anti-MSLN, ${ }^{111}$ In-MORAb009-CHX-A, ${ }^{89} \mathrm{Zr}$-MMOT0530A, ${ }^{111} \mathrm{In}$-amatuximab, ${ }^{99 \mathrm{~m}} \mathrm{Tc}$ A1, ${ }^{89} \mathrm{Zr}$-AMA, ${ }^{89} \mathrm{Zr}$-amatuximab, ${ }^{64} \mathrm{Cu}$-amatuximab, ${ }^{89} \mathrm{Zr}$ labeled MMOT0530A, and ${ }^{89} \mathrm{Zr}$-B3 are the main compounds designed. SPIONs with A-MSF, instead, have been proposed as the T2 targeting agent in pancreatic cancer in MRI. ${ }^{111}$ InmAbMB-SPIONs, indeed, was an exciting proposal as SPECT/MRI probe to detect MSLN positive cancers. Bb-SA-SPIONs are recent applications of nanotechnology developed to obtain a chemical-based strategy that does not interfere with the antibody site of binding of immunotargeted nanoparticles. All the tracers showed specific tumor uptake, while ${ }^{89} \mathrm{Zr}$-labeled MMOT0530A could be used also to predict if a selected patient suits the DMOT4039A ADC treatment. Thus, radiolabeled Ab anti-MSLN could be crucial not only as a treatment tool but it could also be convenient in radioimmunotherapy. An example is M-PLDUs that demonstrated to be a valid vehicle anticancer drugs and a promising contrast agents for pancreatic cancer cells. The assessment of the expression grade of MSLN malignancies gives the possibility to predict the eligibility and the response of a single patient to radioimmunotherapy. It could be of primary importance for developing target therapy and, as a consequence, to be more specific in properly selecting the eligible patients for antibody-drug conjugate treatment.

Acknowledgements Open access funding provided by Universitá degli Studi di Roma La Sapienza within the CRUI-CARE Agreement.

Author contributions MC: Literature search, Literature review, and Manuscript Writing. VF: Manuscript Writing, Editing and Content planning. AM: Literature search, Literature review. GDV: Editing and Content planning.

Funding No funding has been received for this paper.

\section{Compliance with ethical standards}

Conflict of interest The authors declare that they have no conflict of interest.

Ethical standards This article does not contain any studies with human participants or animal subjects performed by any of the authors.

Open Access This article is licensed under a Creative Commons Attribution 4.0 International License, which permits use, sharing, adaptation, distribution and reproduction in any medium or format, as long as you give appropriate credit to the original author(s) and the source, provide a link to the Creative Commons licence, and indicate if changes were made. The images or other third party material in this article are included in the article's Creative Commons licence, unless indicated otherwise in a credit line to the material. If material is not included in the article's Creative Commons licence and your intended use is not permitted by statutory regulation or exceeds the permitted use, you will need to obtain permission directly from the copyright holder. To view a copy of this licence, visit http://creativecommons.org/licenses/by/4.0/.

\section{References}

1. Ansari MO, Ahmad MF, Shadab GGHA et al (2018) Superparamagnetic iron oxide nanoparticles based cancer theranostics: a double edge sword to fight against cancer. J Drug Deliv Sci Technol 45:177-183

2. Argani P, Iacobuzio-Donahue C, Ryu B et al (2001) Mesothelin is overexpressed in the vast majority of ductal adenocarcinomas of the pancreas: identification of a new pancreatic cancer marker by serial analysis of gene expression (SAGE). Clin Cancer Res 7:3862-3868

3. Baldo P, Cecco S (2017) Amatuximab and novel agents targeting mesothelin for solid tumors. Onco Targets Ther 10:5337-5353

4. Bon GG, Kenemans P, Verstraeten R et al (1996) Serum tumor marker immunoassays in gynecologic oncology: establishment of reference values. Am J Obstet Gynecol 174:107-114

5. Chang K, Pai LH, Batra JK et al (1992) Characterization of the antigen (CAK1) recognized by monoclonal antibody K1 present on ovarian cancers and normal mesothelium. Cancer Res 52:181-186

6. Chen SH, Hung WC, Wang P et al (2013) Mesothelin binding to CA125/MUC16 promotes pancreatic cancer cell motility and invasion via MMP-7 activation. Sci Rep 3:1870

7. Cheng WF, Huang CY, Chang MC et al (2009) High mesothelin correlates with chemoresistance and poor survival in epithelial ovarian carcinoma. Br J Cancer 100:1144-1153

8. De Vincentis G, Follacchio GA, Frantellizzi V et al (2016) Prostate-specific antigen flare phenomenon during ${ }^{223} \mathrm{Ra}$-dichloride treatment for bone metastatic castration-resistant prostate cancer: a case report. Clin Genitourin Cancer 14:e529-e533

9. De Vincentis G, Follacchio GA, Frantellizzi V et al (2017) ${ }^{223} \mathrm{Ra}-$ dichloride therapy in an elderly bone metastatic castration-resistant prostate cancer patient: a case report presentation and comparison with existing literature. Aging Clin Exp Res 30:677-680

10. De Vincentis G, Gerritsen W, Gschwend JE et al (2019) Advances in targeted alpha therapy for prostate cancer. Ann Oncol 30:1728-1739

11. Deng L, Ke X, He Z et al (2012) A MSLN-targeted multifunctional nanoimmunoliposome for MRI and targeting therapy in pancreatic cancer. Int J Nanomed 7:5053-5065

12. Elias A, Tsourkas A (2009) Imaging circulating cells and lymphoid tissues with iron oxide nanoparticles. Am Soc Hematol Educ Progr 1:720-726

13. Follacchio GA, Monteleone F, Anibaldi P et al (2015) A modified sentinel node and occult lesion localization (SNOLL) technique in non-palpable breast cancer: a pilot study. J Exp Clin Cancer Res 34:113-113

14. Frantellizzi V, Conte M, Pontico M et al (2020) New frontiers in molecular imaging with Super Paramagnetic Iron Oxide Nanoparticles (SPIONs): efficacy, toxicity, and future applications. Nucl Med Mol Imaging 54:65-80

15. Frantellizzi V, Cosma L, Brunotti G et al (2020) Target alpha therapy with thorium-227. Cancer Biother Radiopharm. https:// doi.org/10.1089/cbr.2019.3105 
16. Gebhart G, Lamberts LE, Wimana Z et al (2016) Molecular imaging as a tool to investigate heterogeneity of advanced HER2-positive breast cancer and to predict patient outcome under trastuzumab emtansine (T-DM1): the ZEPHIR trial. Ann Oncol 27:619-624

17. Hagemann UB, Ellingsen C, Schuhmacher J et al (2019) Mesothelin-targeted thorium-227 conjugate (MSLN-TTC): preclinical evaluation of a new targeted alpha therapy for mesothelin-positive cancers. Clin Cancer Res 25:4723-4734

18. Hassan R, Bera T, Pastan I (2004) Mesothelin: a new target for immunotherapy. Clin Cancer Res 10:3937-3942

19. Hassan R, Blumenschein GR Jr, Moore KN et al (2020) Firstin-human, multicenter, phase I dose-escalation and expansion study of anti-mesothelin antibody-drug conjugate anetumab ravtansine in advanced or metastatic solid tumors. J Clin Oncol 38:1824-1835

20. Hassan R, Bullock S, Premkumar A et al (2007) Phase I study of SS1P, a recombinant anti-mesothelin immunotoxin given as a bolus I.V. infusion to patients with mesothelin-expressing mesothelioma, ovarian, and pancreatic cancers. Clin Cancer Res 13:5144-5149

21. Hassan R, Cohen SJ, Phillips M et al (2010) Phase I clinical trial of the chimeric anti-mesothelin monoclonal antibody MORAb009 in patients with mesothelin-expressing cancers. Clin Cancer Res 16:6132-6138

22. Hassan R, Ebel W, Routhier EL et al (2007) Preclinical evaluation of MORAb-009, a chimeric antibody targeting tumor-associated mesothelin. Cancer Immun 7:20

23. Hassan R, Kindler HL, Jahan T et al (2014) Phase II clinical trial of amatuximab, a chimeric antimesothelin antibody with pemetrexed and cisplatin in advanced unresectable pleural mesothelioma. Clin Cancer Res 20:5927-5936

24. Hassan R, Laszik ZG, Lerner M et al (2005) Mesothelin is overexpressed in pancreaticobiliary adenocarcinomas but not in normal pancreas and chronic pancreatitis. Am J Clin Pathol 124:838-845

25. Hassan R, Schweizer C, Lu KF et al (2010) Inhibition of mesothelin-CA-125 interaction in patients with mesothelioma by the anti-mesothelin monoclonal antibody MORAb-009: implications for cancer therapy. Lung Cancer 68:455-459

26. Hilliard TS (2018) The impact of mesothelin in the ovarian cancer tumor microenvironment. Cancers (Basel) 10:277

27. Houghton JL, Zeglis BM, Abdel-Atti D et al (2016) Pretargeted immuno-PET of pancreatic cancer: overcoming circulating antigen and internalized antibody to reduce radiation doses. J Nucl Med 57:453-459

28. Kobayashi K, Sasaki T, Takenaka F et al (2015) A novel PET imaging using ${ }^{64} \mathrm{Cu}$-labeled monoclonal antibody against mesothelin commonly expressed on cancer cells. J Immunol Res 2015:268172

29. Koller M, Hartmans E, De Groot DJA et al (2017) Data-driven prioritization and review of targets for molecular-based theranostic approaches in pancreatic cancer. J Nucl Med 58:1899-1903

30. Lamberts LE, De Groot DJ, Bense RD et al (2015) Functional genomic mRNA profiling of a large cancer data base demonstrates mesothelin overexpression in a broad range of tumor types. Oncotarget 6:28164-28172

31. Lamberts LE, Der Houven M-V, Van Oordt CW, Ter Weele EJ et al (2016) ImmunoPET with anti-mesothelin antibody in patients with pancreatic and ovarian cancer before anti-mesothelin antibody-drug conjugate treatment. Clin Cancer Res 22:1642-1652

32. Lee JH, Kim H, Yao Z et al (2015) Tumor and organ uptake of (64)Cu-labeled MORAb-009 (amatuximab), an anti-mesothelin antibody, by PET imaging and biodistribution studies. Nucl Med Biol 42:880-886

33. Lee JH, Kim H, Yao Z et al (2018) Tumor-shed antigen affects antibody tumor targeting: comparison of two (89)Zr-labeled antibodies directed against shed or nonshed antigens. Contrast Media Mol Imaging 2018:2461257

34. Lindenberg L, Thomas A, Adler S et al (2015) Safety and biodistribution of $111 \mathrm{In}$-amatuximab in patients with mesothelin expressing cancers using single photon emission computed tomography-computed tomography (SPECT-CT) imaging. Oncotarget 6:4496-4504

35. Liu F, Le W, Mei T et al (2016) In vitro and in vivo targeting imaging of pancreatic cancer using a $\mathrm{Fe}_{3} \mathrm{O}_{4} @ \mathrm{SiO}_{2}$ nanoprobe modified with anti-mesothelin antibody. Int J Nanomed 11:2195-2207

36. Misri R, Meier D, Yung AC et al (2012) Development and evaluation of a dual-modality (MRI/SPECT) molecular imaging bioprobe. Nanomedicine 8:1007-1016

37. Misri R, Saatchi K, Ng SS et al (2011) Evaluation of (111)In labeled antibodies for SPECT imaging of mesothelin expressing tumors. Nucl Med Biol 38:885-896

38. Montemagno C, Cassim S, Trichanh D et al (2019) (99m)Tc-A1 as a novel imaging agent targeting mesothelin-expressing pancreatic ductal adenocarcinoma. Cancers (Basel) 11:1531

39. Ordóñez NG (2003) Application of mesothelin immunostaining in tumor diagnosis. Am J Surg Pathol 27:1418-1428

40. Orth M, Metzger P, Gerum S et al (2019) Pancreatic ductal adenocarcinoma: biological hallmarks, current status, and future perspectives of combined modality treatment approaches. Radiat Oncol 14:141

41. Pani R, Pellegrini R, Bennati P et al (2008) A novel parallel hole collimator for high resolution SPET imaging with a compact $\mathrm{LaBr} 3$ gamma camera. In: IEEE nuclear science symposium conference record, pp 3824-3828

42. Pastan I, Hassan R (2014) Discovery of mesothelin and exploiting it as a target for immunotherapy. Cancer Res 74:2907-2912

43. Peters WA 3rd, Bagley CM, Smith MR (1986) CA-125. Use as a tumor marker with mixed mesodermal tumors of the female genital tract. Cancer 58:2625-2627

44. Pimm MV (1995) Circulating antigen: bad or good for immunoscintigraphy? Nucl Med Biol 22:137-145

45. Pimm MV, Durrant LG, Baldwin RW (1989) Influence of circulating antigen on the biodistribution and tumour localization of radiolabelled monoclonal antibody in a human tumour: nude mouse xenograft model. Eur J Cancer Clin Oncol 25:1325-1332

46. Prantner AM, Nguyen CV, Scholler N (2013) Facile immunotargeting of nanoparticles against tumor antigens using sitespecific biotinylated antibody fragments. J Biomed Nanotechnol 9:1686-1697

47. Quante AS, Ming C, Rottmann M et al (2016) Projections of cancer incidence and cancer-related deaths in Germany by 2020 and 2030. Cancer Med 5:2649-2656

48. Rahib L, Smith BD, Aizenberg R et al (2014) Projecting cancer incidence and deaths to 2030: the unexpected burden of thyroid, liver, and pancreas cancers in the United States. Cancer Res 74:2913-2921

49. Ricci M, Frantellizzi V, Bulzonetti N et al (2018) Reversibility of castration resistance status after radium-223 dichloride treatment: clinical evidence and review of the literature. Int J Radiat Biol 95:554-561

50. Rump A, Morikawa Y, Tanaka M et al (2004) Binding of ovarian cancer antigen CA125/MUC16 to mesothelin mediates cell adhesion. J Biol Chem 279:9190-9198

51. Shin IS, Lee SM, Kim HS et al (2011) Effect of chelator conjugation level and injection dose on tumor and organ uptake of 111Inlabeled MORAb-009, an anti-mesothelin antibody. Nucl Med Biol 38:1119-1127

52. Siegel RL, Miller KD, Jemal A (2018) Cancer statistics, 2018. CA Cancer J Clin 68:7-30

53. Sirois AR, Deny DA, Li Y et al (2020) Engineered Fn3 protein has targeted therapeutic effect on mesothelin-expressing cancer cells 
and increases tumor cell sensitivity to chemotherapy. Biotechnol Bioeng 117:330-341

54. Smith-Jones PM, Pandit-Taskar N, Cao W et al (2008) Preclinical radioimmunotargeting of folate receptor alpha using the monoclonal antibody conjugate DOTA-MORAb-003. Nucl Med Biol 35:343-351

55. Ter Weele EJ, Terwisscha Van Scheltinga AG, Kosterink JG et al (2015) Imaging the distribution of an antibody-drug conjugate constituent targeting mesothelin with ${ }^{89} \mathrm{Zr}$ and IRDye $800 \mathrm{CW}$ in mice bearing human pancreatic tumor xenografts. Oncotarget 6:42081-42090

56. Terwisscha Van Scheltinga AG, Ogasawara A, Pacheco G et al (2017) Preclinical efficacy of an antibody-drug conjugate targeting mesothelin correlates with quantitative 89Zr-immunoPET. Mol Cancer Ther 16:134-142

57. Thomas AM, Santarsiero LM, Lutz ER et al (2004) Mesothelinspecific CD8(+) $\mathrm{T}$ cell responses provide evidence of in vivo cross-priming by antigen-presenting cells in vaccinated pancreatic cancer patients. J Exp Med 200:297-306

58. Yildiz Y, Kabadayi G, Yigit S et al (2019) High expression of mesothelin in advanced serous ovarian cancer is associated with poor prognosis. J Buon 24:1549-1554

59. Yin BW, Lloyd KO (2001) Molecular cloning of the CA125 ovarian cancer antigen: identification as a new mucin, MUC16. J Biol Chem 276:27371-27375

Publisher's Note Springer Nature remains neutral with regard to jurisdictional claims in published maps and institutional affiliations. 